\title{
RENOUVEAU CASSIRERIEN
}

Ernst Cassirer a longtemps fait partie de ces philosophes peu repris par leurs pairs, guère étudiés et insuffisamment traduits ${ }^{1}$. Cette situation est en pleine mutation depuis 1988. D'une part, de nombreux textes sont édités et traduits, d'autre part, études et colloques se multiplient.

Les éditions F. Meiner, en collaboration avec John M. Krois, ont entrepris la publication d'importants inédits, comme le quatrième tome de la Philosophie des formes symboliques. Les traductions françaises s'appliquent à faire paraître les œuvres disponibles en allemand, de même que certains textes inédits. Ainsi $L e$ Mythe de l'État et un Hegel inédit doivent paraître chez Gallimard, ainsi qu'un manuscrit intitulé Sur les types fondamentaux d'anthropologie philosophique aux éditions du Seuil. Les éditions du Cerf poursuivent un remarquable effort puisqu'elles ont notamment en cours les traductions suivantes : la totalité de l'Erkenntnisproblem, le Leibniz'System, Zur Einsteinschen Relativitätstheorie, Freiheit und Form, Idee und Gestalt, Determinismus und Indeterminismus in der modernen Physik, le dernier volume, inédit, de la Philosophie des formes symboliques, trois cours inédits : un Kant, un Goethe et un Schiller, deux recueils comprenant des inédits, l'un sur l'art, l'autre sur la philosophie pratique, un volume sur l'école de Marbourg. Le manque de traduction sera donc très largement comblé d'ici quelques années.

Par ailleurs, les colloques se multiplient : un remarquable volume, Über E. Cassirers Philosophie der symbolischen Formen ${ }^{2}$, en témoignait déjà. Le Cerf a publié en 1990 les actes de celui qui eut lieu du 12 au 14 octobre 1988 à Nanterre : Ernst Cassirer, de Marbourg à New York. Itinéraires philosophiques, sous la direction de Jean Seidengart. Du 24 au 27 septembre 1991 s'est tenu, à l'initiative d'Enno Rudolph et de Heinz Wismann, au Forschungsstätte der evangelischen Studiengemeinschaft de Heidelberg, un symposium intitulé Cassirers Beitrag zur Philosophie des 20. Jahrhunderts dont les actes paraitront dans la revue de cet organisme et dans un numéro spécial de la Revue de métaphysique et de morale.

1. Certaines traductions ont parfois de gros défauts : ainsi, Descartes, Corneille, Christine de Suède, Paris, Vrin, 1942, ne contient en fait que des extraits, avec des intitulés faux et une traduction approximative.

2. Francfort-sur-le-Main, Suhrkamp, 1988.

Revue de synthèse : IV S. Nos 1-2, janv.-juin 1992. 
Nous aimerions ici prendre bonne mesure de deux contributions * à ce renouveau.

Dès 1921, Cassirer situait son projet philosophique dans ses rapports avec les sciences de l'esprit ${ }^{3}$. La Philosophie der symbolischen Formen, publiée de 1923 à 1929 concrétise ce projet ${ }^{4}$. Au tout début des années trente, la poursuite de l'idéal philosophique de l'unité de l'Être, tel que le philosophe en déchiffre l'histoire : d'une unité substantielle vers une unité fonctionnelle, a donc reçu une élaboration théorique qui s'enracine dans l'unité de l'activité symbolique de la conscience et se déploie en une diversité systématique de modes : le mythe, le langage, la science. La philosophie de la culture consiste alors en une "morphologie de l'esprit », expression en laquelle « esprit " doit être entendu au double sens du génitif objectif et subjectif : esprit humain se produisant en un monde, lequel constitue son être-là, sa réalisation. Mais cette période est historiquement douloureuse. Avec l'élection de Hitler commencent les exils du philosophe juif et $A u f$ klärer. Pressante est la nécessité de passer du concept théorique de la philosophie à son concept pratique, du concept scolastique au concept cosmique, affirme-t-il en reprenant, dans le Discours inaugural à l'université de Göteborg en $1935^{5}$, une terminologie kantienne. La philosophie de la culture prend dès lors un tout nouveau sens ${ }^{6}$ : elle n'est plus seulement caractérisée comme «morphologie de l'esprit », comme analyse des configurations du " Globus Intellectualis " ou " cosmos de l'esprit $~^{7}$, mais, orientée par « la fin essentielle de la raison humaine ${ }^{8}$, elle est investie par l'intersubjectivité, puisqu'elle s'avère être la constitution dynamique et commune d'un monde, objectivation des sujets, par lequel ceux-ci, en

* Ernst Cassirer, Logique des sciences de la culture. Cinq études trad. de l'allemand par Jean Carpo, collab. Joẻl Gaubert, précédé de « Fondation critique ou fondation herméneutique des sciences de la culture? ", par J. Gaubert. Paris, Cerf, 1991. $15 \times 24,232$ p. (« Passages $)$ ).

Ernst Cassirer : de Marbourg à New York. Itinéraires philosophiques. Actes du colloque de Nanterre, 12-14 oct. 1988. Éd. sous la dir. de Jean SeIDEngarT. Paris, Cerf, 1990. 14,5 × 23,5, 374 p., index, bibliogr. (" Passages ").

3. Der Begriff der symbolischen Formen im Aufbau der Geisteswissenschaften, Bibliothek Warburg, Vorträge 1921-1922, Leipzig, B.G. Teubner, 1923. On se référera avec profit à l'article de Massimo Ferrari, « Das Problem der Geisteswissenschaften in den Schriften Cassirers für die Bibliothek Warburg (1921-1923) ", in Uber E. Cassirers Philosophie der symbolischen Formen, éd. Hans-Jürg BraUN, Helmut HolzHEY, Ernst Wolfgang ORTH, Francfort-sur-le-Main, Suhrkamp, 1988, p. 114-133.

4. Concernant son intime relation avec Das Erkenntnisproblem, cf. M. FerRari, Il Giovane Cassirer e la scuola di Marbourgo, Milan, Franco Angeli, 1988, ainsi que ID., "La genèse de Das Erkenntnisproblem ", in Ernst Cassirer : de Marbourg à New York, op. cit. supra n. *.

5. « Der Begriff der Philosophie als Problem der Philosophie», in Symbol, Myth and Culture. Essays and Lectures of Emst Cassirer, 1935-1945, éd. Donald Phillip Verene, New Haven/Londres, Yale University Press, 1979, p. 58.

6. Ibid., p. 57 : « La philosophie ne peut se contenter de rechercher la forme et la structure de domaines particuliers de la culture, la structure du langage, de l'art, du droit, du mythe et de la religion [...] Lorsque cette question du but et de la signification est appliquée à la totalité de la culture, nous nous tenons à un tournant décisif de l'auto-réflexion philosophique. ")

7. Philosophie des formes symboliques, Paris, Minuit, 1972, t. I, p. 7 ; t. III, p. 55 ; t. I, p. 54.

8. Cf. supra n. 5. 
communiquant, se constituent en tant qu'êtres singuliers et libres. La question de la philosophie n'est plus alors celle, théorique, de l'unité de l'Être ni même celle de l'unité du sujet, mais celle, pratique, de l'unité fonctionnelle de l'homme en tant qu'elle le conduit à sa liberté tant intellectuelle qu'éthique ${ }^{9}$. Un Essai sur l'homme y répondra explicitement.

En 1942, date de publication de Sur la logique des sciences de la culture, Cassirer a donc achevé les œuvres qui établissent les principes théoriques de sa philosophie, mais l'exposé de celle-ci doit prendre une nouvelle orientation et une nouvelle forme. Le texte publié ici trouve sa spécificité à fonder et à articuler ce passage ${ }^{10}$. Il constitue l'aboutissement de deux courants convergents qui véhiculent l'un une réflexion sur la structure systématique de la philosophie des formes symboliques en tant qu'elle répond au problème des sciences de la culture $^{11}$, l'autre toute la réflexion pratique de Cassirer ${ }^{12}$. Sa visée fondamentale n'est pas une logique des sciences de la culture ${ }^{13}$, mais de prolonger l'instauration d'une conception unitaire de l'homme, remise en cause par la scission moderne

9. Un Essai sur l'homme, trad. Norbert Massa, Paris, Minuit, 1975, p. 40.

10. Il ést important de noter, pour éviter de spéculer sur une évolution du philosophe, que le point de vue anthropologique de l'Essai sur l'homme est déjà traité dans la deuxième partie du manuscrit du volume IV de la Philosophie des formes symboliques : « Le problème du symbole comme problème fondamental de l'anthropologie philosophique ", datant de 1928 (inédit). Cf. Josef Maria WerLE, « E. Cassirers nachgelassene Aufzeichnungen uber " Leben und Geist. Zur Kritik der Philosophie der Gegenwart" ", in op. cit. supra n. 2.

11. Outre l'article paru en 1923, on en trouve l'expression dans « Zur Logik des Symbolbegriffs ", Theoria, Göteborg, vol. IV, 1938, p. 145-175, rééd. in Wesen und Wirkung des Symbolbegriffs, Darmstadt, Wissenschaftliche Buchgesellschaft, 1956, puis 1976, et dans "Sur la logique des sciences de l'esprit ", in Äxel Hägerström. Eine Studie zur Schwedischen Philosophie der Gegenwart, Göteborgs Hoggskolas Arsskrift, XLV, 1939, chap. v. Les deux chapitres précédents - " La philosophie morale ", " Droit et mythe » - laissaient clairement apparaître, à travers la dimension polémique avec l'École d'Uppsala, les préoccupations pratiques de Cassirer. De 1939 date également la conférence donnée d'abord à Vienne en 1936 : « Fondation naturaliste et fondation humaniste de la philosophie de la culture ", Goteborgs Kungl. Vetenskaps-och Vitterhets, Samhallets Handlingar, Goteborg, Ser. A, 3, 1939, trad. franç. in L'Idée de l'histoire, Paris, Cerf, 1988. Enfin, de 1939 à 1944, Cassirer avait donné une série de conférences et de cours sur ce sujet, en part. à Göteborg et à Lund, en avril 1941, répertoriée dans le catalogue de ses manuscrits : " On the Epistemology of the Humanistic Sciences ", " Problems of the Philosophy of Culture ", " Basic Problems of the Philosophy of Culture $»$.

12. Outre les textes publiés, précités n. 11, dans le recueil L'Idée de l'histoire et les chapitres du Äxel Hägenström, on doit se référer à Freiheit und Form, Studien zur Geistesgeschichte, Berlin, B. Cassirer, 1916, et à une série d'articles (dont la traduction est en cours chez le même éditeur) : Die Idee der republikanischen Verfassung, Hambourg, Friedrischen, 1929, "Vom Wesen und Werden des Naturrechts", Zeitschrift fir Rechtsphilosophie, vol. VI, Leipzig, Meiner, 1932, " Der Begriff der Philosophie als Problem der Philosophie », in op. cit. supra n. 3. Cette réflexion aboutit aux ouvrages connus Un Essai sur l'homme, op. cit. supra n. 9 et Le Mythe de l'État, à paraître chez Gallimard - ainsi qu'aux articles : « The Technique of our Modern Political Myths ", in Symbol, Myth, and Culture, op. cit. supra n. 5 , p. 242-267, "Judaism and the Modern Political Myths", Contemporary Jewish Record, vol. VII, 1944, "Albert Schweitzer as Critic of Nineteenth-Century Ethics", in The A. Schweitzer Jubilee Book, éd. A. A. Roвack, Cambridge, Sci-art Publishers, 1946.

13. Aussi le titre devrait-il être traduit plus littéralement : «Sur la logique des sciences de la culture $"$. 
entre science de la nature et science de la culture, vers son sens pratique : l'unité intersubjective, médiatisée par la culture (dont la science fait partie), comme réalisation de l'essence de l'homme.

La première étude, l'Objet des sciences de la culture, réaffirme le programme que l'introduction de la Philosophie des formes symboliques déployait: « reprendre à son compte la prétention à l'unité et à l'universalité que la métaphysique, sous sa forme dogmatique, avait dû abandonner" (p. 96). Ainsi cet objet n'est pas défini en opposition à celui des sciences de la nature, mais on recherche leur accord, au sein du tout de la philosophie ${ }^{14}$, par l'unité de l'activité symbolique dont ils procèdent. Il faut alors saisir, au sein de cette unité globale, "la différence spécifique entre "science de la nature" et "science de la culture " " (p. 141). Ayant dégagé deux réquisits pour la naissance d'une réflexion intellectuelle : que l'homme se sente invité et autorisé à poser des questions et qu'il se forge une méthode pour y répondre, Cassirer retrace l'histoire des relations entre la méthode et l'objet circonscrit. Il en ressort que l'unité recherchée se fait par la négation de l'un des termes ou que du moins ils restent irréconciliablement séparés : le sensible s'oppose à l'intelligible, le royaume de la nature à celui de la grâce, la substance étendue à la substance pensante. Leibniz et Kant sont les premières unifications cohérentes; la Scienza Nuova de Vico, bien que restaurant une scission, apparaît comme la première fondation des sciences de la culture. Cet historique de la problématique de l'unité, suivant un trajet parallèle et souvent ancré dans l'Erkenntnisproblem, se porte ensuite sur la période moderne, et examine l'unification que sont susceptibles de produire les concepts d'évolution et de forme organique depuis Darwin et chez Uexküll. L'échec d'une unification du cosmos humain par la biologie apparait alors. La question unitaire "Qu'est-ce que l'Homme? " reste par conséquent aporétique puisqu'il demeure « citoyen de deux mondes". Seule une philosophie du symbole peut parvenir à l'unité en maintenant la diversité non rhapsodique des formes symboliques constitutives du cosmos humain, de la culture : mythe, religion, langage, art, histoire, technique, science.

L'étude Perception de chose et perception de l'expression part de cette crise de l'unité du savoir que l'Essai sur l'homme thématisera comme crise de la connaissance de soi, et modélise les solutions jusque-là déployées : depuis la dernière grande tentative d'unification hégélienne, ce ne sont que des variations sur la philosophie de l'esprit qui ont vu le jour. Les trois paradigmes du savoir moderne sont référés à cette structure spéculative : le psychologisme à l'esprit subjectif, l'historicisme à l'esprit objectif et la métaphysique à l'esprit absolu. Les apparentes solutions critiques que proposent l'École de Bade (Windelband et Ricket) ou Hermann Paul (néo-herbartien) sont respectivement reconduites à la métaphysique et à un historicisme doublé de psychologie. L'origine de l'erreur réside dans le point de départ de l'étude : les formes conceptuelles.

Aussi faut-il remonter jusqu'à la perception, pour en faire une phénoménologie. On y rencontre deux problèmes : 1) celui de la représentation, entendue non

14. Cf. « Fondation naturaliste... ", art. cit. supra n. 11, p. 25. 
pas comme extériorité des choses et des sujets, mais comme symbolisation. Il est de l'essence des premiers principes d'être thétiques, non démonstratifs. La remontée à la perception par la phénoménologie a ce caractère anté-réflexif. La vérification se fait alors a parte pro, en exigeant « que tous les phénomènes portés à la lumière de la réflexion ne montrent plus alors aucune contradiction interne et s'harmonisent entre eux » (p. 126). La suppression de la scission, dans l'unité globale de la philosophie des formes symboliques, est donc la garantie de sa vérité. C'est là un processus de scientificité philosophique explicitement kantien et un réquisit constitutif de l'essence du néo-kantisme ${ }^{15}$. 2) Qu'en est-il de la dualité à laquelle le moi s'oppose, le « ça » et le " toi ». Originairement, ces deux dimensions, bien qu'irréductibles, sont intimement fondues. Dans la perception des choses et celle de l'expression, il s'agit des premiers pas vers ce qui constitue deux " orientations " ou « directions ». La (dé)monstration est double : a) une analyse phénoménologique du contenu, b) une ontogenèse (lors de l'acquisition du langage chez l'enfant, la perception de l'expression est première), et une phylogenèse (la façon dont la science se constitue par un mouvement de négation du phénomène de l'expression). La spécificité du traitement de cette problématique, par rapport à la Philosophie des formes symboliques (vol. III), réside dans l'accentuation du rapport avec autrui, tel que la cinquième étude lui donnera toute son ampleur ${ }^{16}$.

Cassirer peut alors revenir au niveau logique en étant assuré que puisque tout concept doit se nourrir dans l'intuition, à la double orientation de la perception doivent correspondre deux types de concepts. Ceux des sciences de la culture doivent par ailleurs intégrer en une réelle synthèse les abords scientifique, historique et psychologique de l'objet culturel. C'est dans les subsomptions ou les synthèses accomplies par les concepts de forme et de style que Cassirer trouve la structure logique des sciences de la culture en réfutant, par l'examen de la linguistique de Humboldt et l'esthétique de Wölfflin, les points de vue réducteurs de Rickert, de $\mathbf{H}$. Paul et le naturalisme de Taine.

La quatrième étude articule du point de vue des sciences de la culture ce qui semble toujours s'opposer : l'analyse du devenir, s'appuyant sur la catégorie de cause et d'effet, et l'analyse de l'cuvre et celle de la forme. Les trois caractères de l'objet culturel précédemment distingués (p. 124) : son être-là matériel, sa description et la description de l'expression personnelle, produisent donc une triple approche (p. 149) : physique, interprétative selon un versant historique (l'histoire historienne) et selon un versant psychologique (" s'expriment là certaines attitudes spirituelles fondamentales $")$. Ces trois degrés sont intégrés dans une synthèse supérieure : l'analyse de l'œuvre, niveau philosophique fondamental. C'est à son sujet que Cassirer parle d'une herméneutique qui permet que se détachent certaines figures fondamentales et qu'apparaissent certaines classes, relations et règles constitutives de ces classes. On accède alors au niveau suivant constitué,

15. Cf. l'article « Neo-Kantianism », Encyclopaedia britannica, $14^{e}$ éd.

16. Irène KaJON, dans « Das Problem der Einheit des Bewusstseins im Denken E. Cassirers ", in op. cit. supra n. 2, et dans Il Conceto dell'unita della cultura, Rome, Bulzoni, 1984, tente d'éclairer cette problématique. 
d'une part, de l'analyse de la forme en elle-même et dans ses rapports mutuels avec les autres formes, d'autre part, en une analyse de l'agir : des « processus psychiques qui ont fait naitre les formes et dont elles sont la trace objective ". Est ainsi produite une théorie de la culture qui vise comme terme asymptotique - car le concept d'homme est un focus imaginarius - une philosophie des formes symboliques. Le dernier niveau consiste en une analyse du devenir. Il est postérieur à l'analyse de la forme, car il doit examiner comment « l'essence de la forme se transforme et se développe " (p. 192). Il s'agit de l'histoire philosophique.

La dernière étude examine la possibilité pour l'homme d'accomplir par et dans la culture sinon son bonheur, ce que Rousseau et Kant ont déjà réfuté, du moins sa liberté. Selon Simmel, le mouvement de production de la culture engendre de son sein même une scission entre le moi et l'ensemble des œuvres. L'individu se trouve relégué en deçà d'un monde culturel hypostasié qui se constitue en structure autonome, ruinant la possibilité d'une intersubjectivité effective établie par la médiation des cuvres. Loin de nier le mouvement d'objectivation du moi, Cassirer y voit, en amont, puisque le pur moi n'est rien de déterminé, le présupposé pour sa propre constitution et, en aval, considère que «l'on trouve au terme du chemin [...] l'autre sujet qui reçoit cette cuvre pour l'introduire dans sa vie et la reconvertir ainsi en ce milieu d'où elle est initialement sortie » (p. 203). La problématique se dédouble alors : synchroniquement, il s'agit de la communication intersubjective telle qu'elle permet que se constitue la culture d'une époque, diachroniquement se déploie le conflit entre tradition et nouveauté. Le mouvement général du devenir de la culture apparaît alors : non une tragédie en laquelle les opposants succombent, mais le drame de l'esprit, en lequel ses forces se dynamisent réciproquement. Ce devenir n'est pas la transformation de la nature qui fait passer d'un genre à l'autre sans que la particularité de l'individu puisse s'y inscrire en tant que telle, mais il réside en la «formation de l'humanité » (p. 223) : d'une part, la façon dont l'activité des individus, produisant ce monde commun à tous, fait évoluer le genre vers sa réalisation et sa liberté, d'autre part, la culture objective un sensus communis plural qui renvoie à $œ$ que Kant nommait toujours substrat intelligible de l'humanité.

En abordant la philosophie de Cassirer, le lecteur est confronté à une série de problèmes : tout d'abord, il s'agit d'un néo-kantien, élève de Cohen, la question se pose donc de sa lecture de Kant et, corrélativement, des relations qu'il eut avec l'École de Marbourg. Ensuite, l'image trop répandue de Cassirer est double : c'est celle d'une historien ou d'un épistémologue. Force est donc de comprendre le statut de l'histoire et le rapport qu'elle entretient avec sa philosophie. Enfin, il faut non seulement étudier chacune des fonctions symboliques décrites tout au long de l'œuvre, mais aussi saisir leur unité systématique et leur orientation téléologique. Les actes publiés par Jean Seidengart, sous le titre Ernst Cassirer : de Marbourg à New York, tentent d'éclairer ces problèmes.

La genèse de la pensée de Cassirer est l'objet de deux articles : " Ernst Cassirer et l'École de Marbourg ", par D. Bourel ; "La genèse de Das Erkenntnisproblem », par M. Ferrari. Le premier, après avoir signalé que son sujet «a été traité de façon quasi définitive " dans le livre de M. Ferrari, Il Giovane Cassirer e la 
scuola di Marburgo, réussit néanmoins à restituer la richesse d'une ambiance intellectuelle souvent méconnue. Le second, dans sa dimension généalogique, aborde également les filiations avec Marbourg, notamment l'insertion de l'historicité dans le transcendantal et les relations entre l'histoire et le système chez Cohen et Natorp. Mais la genèse ici effectuée est aussi interne, examinant les rapports entre les œuvres dites philosophiques - à cette époque Substance et Fonction et ce qui a trop souvent été perçu comme un livre d'histoire : Le Problème de la connaissance. Il en ressort que ce livre est en fait un ouvrage de philosophie, tant l'intérêt systématique prévaut sur l'intérêt historique.

Concernant le traitement des objets historiques, M. de Gandillac, sans ignorer que Cassirer porte plus son attention sur l'unité spirituelle ${ }^{17}$ qui détermine la pensée d'une époque, circonscrit les absences, voire les distorsions dans le tableau de la Renaissance brossé dans Individu et Cosmos. J.-L. Marion tente, pour sa part, de mettre à jour les impasses herméneutiques auxquelles le néo-kantisme de Cassirer devait le conduire dans son interprétation de Descartes et Leibniz. Enfin deux articles, celui d'Alexis Philonenko, " Cassirer lecteur et interprète de Kant ", et celui de Pierre Aubenque, "Le débat de 1929 entre Cassirer et Heidegger " se confrontent au principal enjeu de l'interprétation cassirerienne de Kant, celui du sens de la finitude humaine.

Les travaux sur la pensée scientifique sont particulièrement bien illustrés: Michel Fichant, dans « E. Cassirer et les commencements de la science classique ", débute en rappelant le statut particulier de l'histoire et dégage, par une analyse scrupuleuse du rôle attribué à Galilée, la pratique d'historien du philosophe et les relations entre l'histoire et la science comme formes symboliques. L'article de J. Seidengart, "Théorie de la connaissance et épistémologie de la physique selon E. Cassirer ", fait clairement apparaître l'évolution de l'articulation entre l'historique et le systématique, le travail de la méthode transcendantale, dans la constitution de la théorie de la connaissance de Cassirer. La communication de J.J. Szczeciniarz applique le même type d'attention à la philosophie cassirerienne des mathématiques.

Outre la pensée scientifique et l'histoire, largement représentées, le mythe, le langage et l'art sont chacun l'objet d'une étude. Pierre Caussat, avec une communication intitulée « Entre Humboldt et le structuralisme », examine la possibilité même de considérer le langage comme une forme symbolique. E. Pinto expose les relations et les influences réciproques qui pourraient avoir lié Cassirer et Aby Warburg.

Eu égard à la configuration actuelle des études cassireriennes, l'apport le plus novateur de ce recueil est dû à l'article d'Irène Kajon, «La philosophie, le judaïsme et le mythe politique moderne chez E. Cassirer ». Justice y est rendu à la dimension pratique - morale et politique - de la pensée de Cassirer, aspect certes apparemment peu présent dans l'œuvre du philosophe mais pourtant fondamental car, comme chez Kant, le théorique se subordonne au pratique qui est

17. Cf. notamment : Individu et cosmos dans la philosophie de la Renaissance, trad. Pierre Quillet, Paris, Minuit, 1983, p. 12; La Philosophie des Lumières, trad. P. Quillet, Paris, Fayard, 1970, p. 31. 
fin suprême de la philosophie dans son concept cosmologique. La communication de M. Tripp, " Mythe, technique et l'état moderne selon E. Cassirer ", prend la même orientation en s'attachant plus spécifiquement, par une comparaison avec $H$. Arendt et F. Neumann, au rôle de la technique.

L'étude de $\mathbf{R}$. Nadeau, "Cassirer et le programme d'une épistémologie comparée $"$, s'intéresse à la totalité du projet cassirerien, pour examiner trois critiques possibles : l'insuffisance de sa théorie du symbole, les lacunes des critères de démarcation entre les différentes formes, le problème des rapports génétiques et normatifs entre les diverses ontologies constitutives de ces formes. La théorie du symbole, point nodal qui assure l'unité systématique des diverses formes symboliques est également abordée par A. Kremer-Marietti.

Enfin, trois articles examinent la structure systématique théorique de la philosophie des formes symboliques : P. Osmo développe les métaphores vitalistes que Cassirer emploie au sujet de la culture, de l'esprit, des formes symboliques et s'interroge sur leur validité pour la compréhension de la dynamique même de sa philosophie. A. Stanguennec analyse les emprunts que Cassirer, tout en restant fidèle à l'esprit de la philosophie critique, fait à Hegel pour constituer la genèse téléologique du système des formes symboliques. P. Quillet dégage, à partir de l'analyse du concept de problème, au sein de la non-clôture caractéristique des systèmes critiques, le style proprement cassirerien d'ouverture.

À juger cet ouvrage au but qu'il s'assigne - « présenter un instrument de travail et de réflexion sur la philosophie de E. Cassirer " - et aux limites qu'il se fixe : " nullement couvrir de façon exhaustive la totalité des questions explorées " par le philosophe, on doit constater son évidente réussite, à laquelle la bibliographie compilée par R. Nadeau apporte un précieux tribut. Bien plus, on peut y trouver l'heureux témoignage d'un nouvel abord, strictement nécessaire, jusqu'à présent peu courant à l'étranger, totalement absent en France.

Fabien Capeillères. 